\title{
Médiévales
}

Langues, Textes, Histoire

51 | automne 2006

L'Occident sur ses marges ( $\mathrm{VI} \mathrm{e}^{\mathrm{e}} \mathrm{X} \mathrm{I}^{\mathrm{e}}$ siècles)

\section{John Blair, The Church in Anglo-Saxon Society}

Oxford, Oxford University Press, 2005, 604 p.

\section{Élisabeth Lorans}

\section{(2) OpenEdition}

Journals

Édition électronique

URL : https://journals.openedition.org/medievales/1448

DOI : $10.4000 /$ medievales. 1448

ISSN : $1777-5892$

Éditeur

Presses universitaires de Vincennes

\section{Édition imprimée}

Date de publication : 1 décembre 2006

Pagination : 173-178

ISBN : 978-2-84292-193-4

ISSN : 0751-2708

Référence électronique

Élisabeth Lorans, "John Blair, The Church in Anglo-Saxon Society », Médiévales [En ligne], 51 | automne 2006, mis en ligne le 12 mars 2007, consulté le 23 avril 2022. URL : http://journals.openedition.org/ medievales/1448; DOI : https://doi.org/10.4000/medievales.1448

Ce document a été généré automatiquement le 23 avril 2022.

Tous droits réservés 


\title{
John Blair, The Church in Anglo-Saxon Society
}

Oxford, Oxford University Press, 2005, 604 p.

\author{
Élisabeth Lorans
}

1 L'ouvrage, incluant une abondante bibliographie, un index détaillé et une cinquantaine de figures, que nous offre John Blair constitue le point d'orgue de longues années de recherche consacrées à la place de l'Église et des églises dans la société anglo-saxonne selon une approche qui associe étroitement les sources écrites aux sources matérielles, sous la forme d'analyses topographiques ou de données de fouilles, une démarche qu'avait illustré, dans un registre comparable, le livre de Richard Morris, Churches in the Landscape, il y a plus de quinze ans ${ }^{1}$.

2 Au centre de la réflexion de John Blair, les minsters, définis de manière large comme des communautés religieuses, d'hommes ou de femmes, parfois des deux réunis, moines et/ ou prêtres, placés sous l'autorité d'un abbé, d'une abbesse ou d'un prêtre, et partageant des pratiques liturgiques communes: une diversité de statuts, de modes de vie et de missions qui interdit de traduire le latin "monasterium», qui désigne tous ses établissements dans les sources médiévales, par « monastery» terme suggérant, comme le français "monastère ", une vie régulière, fondée sur une règle unique, telle qu'elle a existé seulement après la Réforme bénédictine, sur le continent comme outre-Manche. Cela conduit l'auteur à utiliser le terme de "minster», dérivé du vieil anglais " mynster», une pratique à laquelle je me conformerai aussi.

3 L'analyse, décomposée en huit chapitres, couvre une période comprise entre le milieu du vi ${ }^{\mathrm{e}}$ siècle et la fin du XI $\mathrm{e}^{\mathrm{e}}$ siècle, un terminus chronologique qui distingue d'emblée cet ouvrage de la majeure partie de la production historique relative à l'Angleterre du haut Moyen Âge qui prend la date de 1066 comme date-buttoir, comme si la conquête normande avait radicalement et rapidement transformé tous les aspects de la société anglaise. Après un chapitre introductif consacré à la situation de l'Angleterre et de ses voisins, insulaires et continentaux, entre 550 et 650 , l'ouvrage est articulé en deux grandes périodes, $650-850$ et 850-1100, qui correspondent respectivement à la période de fondation et d'essor des minsters, puis à un temps de transformations et de 
compétition entre les anciennes institutions et les nouvelles que représentent des églises que l'on pourra pleinement qualifier de paroissiales à l'issue de la période étudiée.

4 L'ouvrage décortique donc les interactions entre l'Église, les églises et la société anglaise, en mettant en évidence les influences extérieures, au premier rang desquelles figurent celles de l'Irlande et du monde franc, et les spécificités de l'Église insulaire. À une Europe méditerranéenne, fortement romanisée et caractérisée par un pouvoir épiscopal bien implanté dans les chefs-lieux de cités, John Blair oppose une Europe septentrionale (qui commence à la Loire), où une organisation politique moins centralisée a laissé place à une autre forme d'organisation ecclésiale, fondée sur un réseau de communautés religieuses et non pas sur une structure hiérarchisée dirigée par les évêques. De ce constat, découle un certain nombre de thèses majeures, qui, toutes, concourent à éclairer le rôle véritablement fondateur des minsters, considérés comme les pivots de la société anglaise pendant sa période même de formation. Sans prétendre rendre compte de toutes les facettes de cette très riche étude, je voudrais aborder les questions ayant trait aux multiples fonctions des minsters et partant à leur rôle dans la structuration de l'espace, principalement entre le viI et le IX ${ }^{e}$ siècle.

5 À la lecture de l'ouvrage de John Blair, les minsters apparaissent en effet comme des pôles multi-fonctionnels qui ont rassemblé les vivants autour d'une grande diversité d'activités mais aussi, de manière progressive, les défunts. Lieux de vie de communautés au statut souvent difficile à cerner, fondés dans un premier temps par les souverains et la grande aristocratie puis par des notables de rang inférieur, grâce à des donations royales, les minsters sont d'abord des centres religieux, non seulement pour ceux qui y résident de manière permanente mais aussi pour toute la population environnante. L'Angleterre des $\mathrm{VII}^{\mathrm{e}}-\mathrm{IX}^{\mathrm{e}}$ siècles se distingue en effet par le caractère relativement centralisé de sa structure ecclésiale : c'est le personnel des minsters qui a assuré l'encadrement pastoral et c'est dans ces établissements que la population laïque recevait les sacrements de l'Église. Sources écrites et sources matérielles concordent ici car bien rares sont les lieux de culte antérieurs au milieu du $\mathrm{x}^{\mathrm{e}}$ siècle, découverts dans les habitats ruraux comme dans les agglomérations, d'origine romaine ou non, hormis les minsters eux-mêmes et leurs dépendances.

6 En l'absence quasi totale d'églises secondaires dans les premiers siècles qui ont suivi la conversion, c'est autour des minsters que peu à peu les morts ont été rassemblés, en milieu rural comme dans les anciens chefs-lieux de cités. En plusieurs étapes, John Blair interroge les pratiques funéraires pour éclairer le passage du paganisme au christianisme, en refusant une opposition tranchée entre les deux formes de vie religieuse, pendant la période de conversion et même avant où l'adoption de nouvelles structures monumentales, illustrée par le site royal et cérémoniel de Yeavering, montre un rapprochement des usages, tout comme les riches inhumations sous tumuli, version païenne de l'inhumation dans les églises prônée par l'aristocratie franque déjà convertie. Sur la question si largement débattue ces dernières années de l'interprétation des pratiques funéraires ${ }^{2}$, John Blair se démarque nettement des interprétations de Martin Carver qui voit dans l'émergence de certains usages, comme les tombes-bateaux richement dotées de Sutton Hoo, la réaction d'une société païenne et orientée vers la Scandinavie à la menace que représenteraient la christianisation et l'influence franque grandissante dans le sud de l'Angleterre ${ }^{3}$. De même, alors que les travaux les plus récents insistent sur les causes culturelles et économiques pour 
expliquer la disparition du mobilier funéraire ${ }^{4}$, l'auteur, tout en acceptant que l'affaiblissement de cette pratique au début $\mathrm{du} \mathrm{VII}^{\mathrm{e}}$ siècle résulte de changements sociaux, voit dans sa disparition totale un siècle plus tard la marque du succès des modes d'inhumation chrétiens: désormais l'identité chrétienne est exprimée par l'usage du linceul et du cercueil, même si à cette date elle ne passe pas encore systématiquement par une inhumation dans un enclos ecclésial. Si les cimetières fondés auprès des minsters ont accueilli non seulement les membres de la communauté mais aussi des laïcs, dans le courant du viI ${ }^{\mathrm{e}}$ siècle, ce mouvement n'a d'abord touché qu'une faible part de la population, probablement les notables, protecteurs de ces établissements, et leurs dépendants avant de gagner du terrain, les petites nécropoles, dépourvues de lieux de culte, étant restées en usage au moins jusqu'aux alentours de 900. En effet, contrairement à ce qui est largement constaté dans l'espace franc et audelà, la conversion des élites anglo-saxonnes ne s'est presque jamais traduite par la construction de chapelles familiales au sein de nécropoles pré-existantes: une large partie de la population chrétienne a donc été inhumée du $\mathrm{VII}^{\mathrm{e}}$ au $\mathrm{IX}^{\mathrm{e}}$ siècle dans les sites funéraires traditionnels où l'adoption de la nouvelle foi était peu visible, le maintien de la sépulture habillée n'étant nullement preuve de paganisme.

7 Lieux de séjour permanents de communautés dont la majorité des membres appartenait à l'aristocratie, les minsters étaient aussi des centres de vie intellectuelle et artistique comme en témoignent les sources écrites mais aussi nombre de découvertes archéologiques: la quantité et la qualité du mobilier recueilli, qui associe de petits objets tels que des épingles, des stylets, des fermoirs de livres ou de coffrets, à de la vaisselle importée en verre et en céramique ou encore à des monnaies, distinguent les minsters des autres habitats du VIII ${ }^{\mathrm{e}}$ siècle, où seuls les wics présentent une diversité mobilière comparable. Ce constat conduit John Blair à poser la question de l'identification de certains habitats ayant livré une panoplie similaire à des sites monastiques, en l'absence de toute mention textuelle. Ce débat fut d'abord ouvert par l'auteur à propos du hall de Northampton, interprété par le fouilleur comme un palais royal mais identifié par John Blair à un site ecclésiastique composé d'une grande salle, faisant office de réfectoire, encadrée de deux églises, l'ensemble étant presque aligné sur un même axe conformément à un usage très fréquent dans les minsters du haut Moyen Âge ${ }^{5}$. Depuis cette première interrogation, le débat a été notamment nourri par la fouille du site de Flixborough qui a révélé une occupation continue à partir du $\mathrm{VII}^{\mathrm{e}}$ siècle sous la forme principale d'un ensemble de bâtiments sur poteaux associé à un cimetière, très partiellement examiné, et où une zone de rejets a livré un mobilier exceptionnel pour la fin $d u \mathrm{IX}^{\mathrm{e}}$ siècle et le début du $\mathrm{x}^{\mathrm{e}}$ siècle, notamment 21 stylets permettant d'écrire sur des tablettes de cire (soit le quart du total connu en Angleterre à ce jour) : habitat séculier selon Christopher Loveluck ${ }^{6}$, minster selon John Blair qui d'une manière générale affirme le caractère monastique des sites qui présentent la plus grande stabilité (dans le temps et dans l'espace) et la plus grande diversité fonctionnelle démontrée par la richesse du mobilier mis au jour. Ainsi, les minsters, notamment ceux de la côte est de l'Angleterre, seraient aussi des centres de production et d'échange, mieux insérés dans l'économie régionale que les wics dont le rôle majeur serait lié au commerce maritime, selon la thèse traditionnelle qu'adopte ici John Blair ${ }^{7}$. Ce débat pose donc la question de la définition de critères de nature matérielle pour l'identification du statut de sites que les textes ne documentent pas et invite notamment à réfléchir sur le sens à donner aux objets qui sont signes de "literacy » ou 
encore au mobilier considéré comme luxueux ou rare dans le courant du haut Moyen Âge.

Opposant les minsters aux villae royales dans lesquelles les souverains ne résident que de manière intermittente, John Blair voit donc dans les premiers les seuls centres de pouvoir stables jusqu'au IX ${ }^{\mathrm{e}}$ siècle, leur établissement dans une ancienne cité d'origine romaine ayant toujours précédé l'implantation d'un palais royal : une thèse fondée sur une analyse minutieuse des sources textuelles et archéologiques et qui s'oppose au point de vue jusque là prédominant développé en premier lieu par Martin Biddle pour Winchester où, conformément au schéma développé par Carl-Richard Brühl pour les cités de la Gaule, est supposée une continuité du pouvoir politique entre le $\mathrm{v}^{\mathrm{e}}$ et le VII siècle qui aurait vu la fondation d'un palais accompagné d'une église devenue peu après siège épiscopal ${ }^{8}$. À l'inverse, John Blair affirme donc l'antériorité et la prééminence des établissements ecclésiastiques dans la reconquête des anciens sites romains, villes, forts ou villae, souvent offerts par les souverains eux-mêmes pour la fondation d'un minster, une étape importante dans la construction d'une identité romaine par des autorités religieuses d'origine anglaise, passés les premiers temps de la conversion ${ }^{9}$.

Définis comme des centres caractérisés par des activités d'une grande diversité qui en font pour l'auteur des "proto-villes", dans un monde où les anciens chefs-lieux romains n'avaient plus, selon la thèse prédominante en Angleterre, de fonction urbaine ${ }^{10}$, les minsters sont considérés comme étant à l'origine de la territorialisation de l'espace. Leur rôle dans la formation du réseau paroissial a fait l'objet d'un débat animé qui a opposé au milieu des années 1990 les tenants de l'ancienneté du maillage, qui aurait été fondé sur des paroisses-mères que de nombreuses études régionales ont tenté de restituer à partir des redevances attestées vers 1100 , à ceux qui voyaient dans les paroisses fragmentées dans le courant du XII ${ }^{\mathrm{e}}$ siècle des territoires formés seulement au $\mathrm{x}^{\mathrm{e}}$ siècle ${ }^{11}$. John Blair, dix ans plus tard, souscrit encore à la première position, tout en l'ayant nuancée pour adopter une voie moyenne: le $\mathrm{x}^{\mathrm{e}}$ siècle aurait vu une réorganisation fondamentale des territoires mais sur la base de structures «quasi paroissiales » plus anciennes, si l'on se fie à la forte corrélation mise en évidence par de récentes études micro-régionales entre paroisses et centaines. D'une manière générale, l'auteur affirme la capacité des élites du haut Moyen Âge, tant laïques qu'ecclésiastiques, à découper le territoire à des fins de gestion, ce que prouveraient les donations de terres. On peut toutefois souligner la différence qui existe entre le découpage (partiel) du territoire en unités d'exploitation et/ou de redevance et la réalisation d'un maillage continu à l'échelle de vastes diocèses, qui plus est en l'absence d'un réseau d'églises secondaires. La position de John Blair parait comparable à celle de Michel Aubrun qui a proposé pour la France des vagues de fondations paroissiales par découpage successif des territoires initiaux, avec comme postulat la délimitation précoce des contours, un shéma que l'enquête menée à l'échelle du diocèse de Tours par Élisabeth Zadora-Rio conduit à remettre en cause.

10 À l'inverse, les sources écrites suggèrent le caractère tardif de la délimitation du droit d'asile, qui n'est attesté en Angleterre qu'au $\mathrm{x}^{\mathrm{e}}$ siècle: des zones de protection légalement définies n'étaient donc pas des éléments importants de la topographie chrétienne de l'Angleterre pendant l'essentiel du haut Moyen Âge et leur développement traduirait la territorialisation tardive d'un privilège laïc en milieu ecclésiastique ${ }^{12}$. 
11 l'année, auraient entrâné une plus forte pression sur leurs terres que sur celles relevant des villae royales, conférant ainsi aux minsters un rôle majeur dans la genèse de la seigneurie, une seigneurie que John Blair qualifie d'extensive. L'Église aurait seule été capable de dégager des surplus, notamment en grains, et aurait favorisé des innovations technologiques telles que les moulins à roue horizontale.

12

Fondée sur la (re)lecture d'une masse considérable de sources, tant écrites que matérielles, cet ouvrage passe en revue toutes les structures de la société pour faire ressortir les champs d'action des communautés religieuses fondées en si grand nombre dans les différents royaumes anglo-saxons, où elles ont souvent constitué des instruments majeurs des politiques dynastiques. Centres de vie religieuse insérés dans l'Église universelle par leur topographie, le choix des dédicaces et leurs pratiques liturgiques, les minsters représentent jusqu'au $\mathrm{IX}^{\mathrm{e}}$ siècle au moins, si l'on suit John Blair, les habitats les plus développés et les plus stables, les plus riches aussi. Ils auraient irrigué dans le tissu local non seulement les nouvelles pratiques religieuses mais aussi de nouveaux produits, importés ou fabriqués sur place, stimulant en particulier la production agricole par une demande forte et continue. Finalement, c'est la diversité des processus d'incorporation des vivants et des morts dans la sphère ecclésiale que ce livre donne à voir. En conclusion, il faut regretter, avec l'auteur, la rareté des fouilles archéologiques de grande ampleur qui sont seules à même de révéler l'organisation spatiale de ces sites supposés complexes et de confirmer leurs multiples fonctions tout au long du haut Moyen Âge.

\section{NOTES}

1. R. MORRIS, Churches in the Landscape, Londres, J. M. Dent \& Sons LMT, 1989.

2. Voir notamment S. Lucy et A. REYNOLDS (éd.), Burial in Early Medieval England and Wales, Londres, The Society for Medieval Archaeology, 2002, dont j'ai publié un compte rendu dans Archéologie médiévale, 34, 2004, p. 312-315.

3. Voir M. CARVER, Sutton Hoo: A Seventh-Century Princely Burial Ground and its Context, Londres, British Museum Press, 2005.

4. H. GEAKE, The Use of Grave-Goods in Conversion-Period England, Oxford, BAR, 1997.

5. J. BLAIR, « Palaces or minsters? Northampton and Cheddar reconsidered », AngloSaxon England, 25, 1996, p. 97-121.

6. C. LOVELUCK, « Wealth, waste and conspicuous consumption. Flixborough and its importance for mid and late Saxon settlement studies ", dans H. HAMERow et

A. MACGREGOR (éd.), Image and Power in the Archaeology of Early Medieval Britain. Essays in honour of Rosemary Cramp, Oxford, Oxbow Books, 2001, p. 78-130.

7. Des travaux récents insistent davantage sur la fonction productive des wics que sur leur rôle dans le commerce à longue distance. Voir notamment A. MORTON, « Hamwic in its context », dans M. ANDERTON (éd.), Anglo-Saxon Trading Centres : Beyond the Emporia, Glasgow, Cruithne Press, 1999, p. 48-62; pour un aperçu des positions récentes 
concernant Hamwic et Ipswich, voir E. LoRANS, « Les élites et l'espace urbain : approches archéologique et morphologique (France du Nord et Angleterre, VII ${ }^{\mathrm{e}}-\mathrm{X}^{\mathrm{e}}$ siècles) », dans $\mathrm{Ph}$. DepreuX (éd.), Les élites et leurs espaces : mobilité, rayonnement, domination ( $\mathrm{VI}^{e}-\mathrm{XI}{ }^{e} \mathrm{~s}$.). Actes du colloque de Göttingen (3-5 mars 2005), Turnhout, Brepols, à paraître.

8. Voir notamment M. BIDDLE, "The development of the anglo-saxon town ", dans Topografia Urbana e vita cittadina nell'alto medioevo in Occidente, Spolète, 1973, p. 203-230 ; M. BIDDLE, « The Study of Winchester : Archaeology and History in a British Town, 1961-1983 ", dans E. G. STANLEY (éd.), British Academy papers on Anglo-Saxon England, Oxford, Oxford University Press, 1990, p. 299-341.

9. La relation des sociétés au passé est l'un des thèmes récurrents de cet ouvrage, qui contribue ainsi à un champ de recherche illustré par de nombreuses publications récentes ; voir notamment J.-M. SANSTERRE (éd.), L'autorité du passé dans les sociétés médiévales, Rome, Coll. de l'EFR 333, 2004.

10. La présentation et la discussion de cette question d'une importance majeure dépassent le cadre de ce compte rendu. John Blair s'appuie ici en particulier sur les conclusions de S. T. LOSEBY, « Power and Towns in Late Roman Britain and Early AngloSaxon England », in G. RIPoll et J. M. GURT (éd.), Sedes regiae (ann. 400-800), Barcelone, Reial Acadèmia de Bones Lletres, 2000, p. 319-370.

11. E. CAMBRIDGE et D. Rollason, « Debate : The pastoral organization of the AngloSaxon Church : a review of the "Minster Hypothesis" », Early Medieval Europe, 4-1, 1995, p. 87-104 ; J. BLAIR, « Debate : Ecclesiastical organization and pastoral care in AngloSaxon England », Early Medieval Europe, 4-2, 1995, p. 193-212.

12. Cette analyse, conduite dans le chapitre 4 (p. 221-228), est d'une lecture quelque peu difficile en l'absence d'une nette distinction entre immunité et droit d'asile.

L'auteur parle de «sacred space » et de « protected space » mais aussi d'« immunities». 